\title{
A New Method for Quick Determination of Molecular Orientation in Poly(ethylene terephthalate) Films by Use of Polarized Microwaves
}

\author{
Shigeyoshi OSAKI \\ Research \& Development, Kanzaki Paper Mfg. Co., Ltd., \\ Amagasaki, Hyogo 660, Japan
}

(Received October 30, 1986)

\begin{abstract}
A new polarized microwave method for determining the orientation of polymer molecules in films without contact and its application to poly(ethylene terephthalate) (PET) are presented. The angular dependence of transmitted microwave intensity allowed the orientation angle, the maximum to minimum intensity ratio, and the ratio of intensities in the transverse to the machine direction to be determined in as a short time as $c a .30 \mathrm{~s}$. The maximum to minimum intensity ratio for uniaxially stretched PET increased with increasing draw ratio. The unstretched and uniaxially and biaxially stretched PET showed orientation patterns different in the angular dependence of transmitted microwave intensity. With the results obtained from X-ray diffraction, mechanical breaking strength, infrared absorption, refractive index, and thermal shrinkage measurements, it was concluded that the orientation angle and the maximum to minimum ratio determined by the microwave method reflect, respectively, the direction and the degree of the orientation of PET chains. Biaxially stretched PET gave different orientation patterns at different positions in the transverse direction. It was found that results from the microwave method give information on the dielectric anisotropy of films and depend on machine condition in preparing
\end{abstract} films.

\author{
KEY WORDS Polarized Microwave / Molecular Orientation / \\ Poly(ethylene terephthalate) / Orientation Angle / Orientation Pattern / \\ Quick Determination / Dielectric Anisotropy / Dimensional Stability /
}

Experimental determination of the orientation of polymer molecules in films is a subject of great importance, since it is indispensable in understanding the relationship between the structure and properties of the polymer.

The molecular orientation has been investigated by several techniques such as X-ray diffraction, infrared dichroism, birefringence, polarized fluorescence, ultrasonic velocity, mechanical breaking strength, and Young's modulus methods. ${ }^{1,2}$ Especially, X-ray diffraction and infrared dichroism have played a very important role for making clear the molecular structure and the molecular orientation. These methods require a long time for determining the degree of molecular orientation and have some other disadvantages: for example, X-ray diffraction reflects an orientation of polymer chains only in a very limited region of the crystal, infrared dichroism is useful only for very thin and transparent films, and birefringence is not applicable to very thin or opaque films. Other methods are not so useful and convenient either; the polarized fluorescence method, which needs a long time for sample preparation, is not applicable to usual films, the ultrasonic method is difficult to obtain accurate data because of ambiguity arising from contact between the piezoelectric element and the surface of sheet, the mechanical breaking strength method does not always give reproducible data because of the de- 
struction of sample and Young's modulus also needs a long time to measure. Thus, it has long been desired to find a method and an apparatus for determining the molecular orientation conveniently and quickly.

For this purpose, we have developed a new method, which utilizes polarized microwaves and is free from any contact and destruction of sample sheets. This method allows the molecular orientation to be determined in as little time as $c a$. $30 \mathrm{~s}$. In terms of accuracy, precision, and rapidity of measurements, the method lends itself to measure a number of samples. In a previous paper, ${ }^{3}$ the fiber orientation of paper sheet determined by means of microwaves was reported. The present paper describes the principle of this method and the determination of molecular orientation in poly(ethylene terephthalate) films.

\section{PRINCIPLE}

For high polymers, there have been observed, in general, a few or more kinds of dielectric relaxation designated $\alpha, \beta, \gamma$, and so on from the high temperature side. ${ }^{4}$ The relaxations $\alpha$ and $\beta(\gamma)$ were assigned, respectively, to the micro-Brownian motion of long flexible dipoles and to local motions such as twist or rotation of short dipoles attached to the polymer chains. ${ }^{4.5}$

Only a few fundamental studies ${ }^{6,7}$ have been reported on dielectric relaxations at microwave frequencies because of a technical problem, though many studies have been made at frequencies below $10 \mathrm{MHz} .^{4,5,8,9-12}$

The microwave region $(300 \mathrm{MHz}-30 \mathrm{GHz})$ corresponds to that of the frequencies at which the dielectric relaxations ascribable mainly to the local motion should be observed at room temperature..$^{8,11}$ It is well known that the dielectric anisotropy due to local motions reflects the molecular orientation of polymer chains. ${ }^{12}$ This anisotropy, which depends on the orientation of molecular chains, may be determined from the angular dependence of interaction between polarized microwaves and the material. The interaction, which can be detected as change in the intensity of the transmitted microwave, depends on the rotating angle of the anisotropic sheet material. The dielectric properties (dielectric constant and dielectric loss) of the sheet are measured in the cavity resonator system and can be expressed as a resonance curve. If the frequency of the polarized microwave which is perpendicularly irradiated to the sample set between a pair of the rectangular cavities is swept, the resonance curve of transmitted microwave intensity $v s$. frequency is obtained. This curve can be described

$$
\begin{aligned}
& I(\theta) / I_{0}(\theta) \\
& \quad=1 /\left[1+Q^{2}(\theta)\left(\omega_{0}(\theta) / \omega(\theta)-\omega(\theta) / \omega_{0}(\theta)\right)^{2}\right]
\end{aligned}
$$

where $I, I_{0}, \omega, \omega_{0}, Q$, and $\theta$ are the transmitted microwave intensity at a given frequency, the transmitted microwave intensity at the resonance frequency, the angular frequency, the resonance angular frequency, the ratio of the resonance angular frequency $\omega_{0}$ to the half width $\Delta \omega$ in the resonance curve, and rotating angle, respectively. The resonance frequency shifts depending on the capacity part of dielectrics, while the change in intensity at the resonance peak depends on that in dielectric loss. Therefore, the anisotropy in the dielectric constant and dielectric loss of the sheet material gives the different resonance curves at different directions in the sheet plane, respectively. In an actual experiment, transmitted microwave intensities are measured at 360 different $\theta$ at a fixed frequency above the resonance frequency.

\section{EXPERIMENTAL}

The apparatus used in the present study was MICROWAVE MOELCULAR ORIENTATION ANALYZER MOA-2001A developed by Kanzaki Paper Mfg. Co., Ltd. ${ }^{3}$ Its block diagram is shown in Figure 1. The micro- 


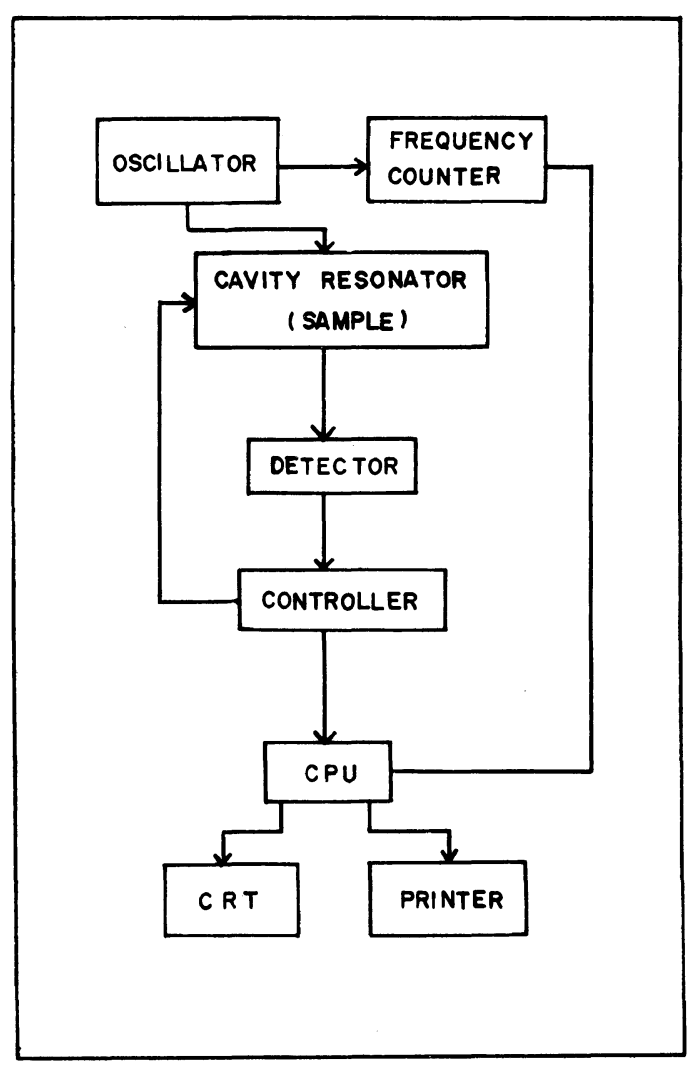

Figure 1. Block diagram of "MICROWAVE MOLECULAR ORIENTATION ANALYZER MOA2001A."

wave cavity resonator system consists of a pair of rectangular waveguides with a narrow free space in which the sample film is set. ${ }^{3}$ The size of the waveguides was determined so that the magnitude of the electric field exited by the oscillator had a maximum at the sample position. The mode of the electromagnetic field was $\mathrm{TE}_{10 \mathrm{~L}} \cdot{ }^{13}$ The sample set between the waveguides in the resonance cavity system was rotated for $6.7 \mathrm{~s}$ around its central axis normal to the sheet plane, and during this period the intensity of the polarized microwaves transmitted through the sample was measured by the detector at 360 points per rotation.

The controller part regulates the rotating angle. The data accumulated in CPU give not only orientation patterns corresponding to the angular dependence of transmitted microwave intensity but also an orientation angle between the main axis of molecular chains and a fixed standard direction, the observed maximum and minimum intensities, and the ratio $I_{\max } /$ $I_{\min }$ of the maximum to minimum intensities. Since the ratio $I_{\max } / I_{\min }$ depends on the sample thickness, a comparison between the ratios at a constant thickness is of meaning for samples with different thicknesses. This ratio should be a measure of the orientation distribution of molecular chains for the sample with almost the same thickness. Further, the ratio of the intensities in TD (the transverse direction perpendicular to the machine direction) to MD (the machine direction or the stretching direction) calculated are displayed on the CRT and recorded on a chart. The data processing time is about $2 \mathrm{~s}$.

The experimental procedure is as follows: The test sample, cut to a fixed size, is set at a standard direction in the cavity resonator system, the frequency is adjusted above the resonance frequency by a dial, and the measurement is made for the computer key board.

The samples used in the present study were poly(ethylene terephthalate) (PET) films. The standard sample size was $100 \mathrm{~mm} \times 100 \mathrm{~mm}$. The 10 different sheet samples $100 \mathrm{~mm}$ in width are cut out of the biaxially stretched film of $1200 \mathrm{~mm}$ in width along the transverse direction TD. The samples were numbered 1 to 10 from left to right side along the TD, as shown in Figures 6 and 7.

The mechanical breaking strength was measured for a PET film with a size of $15 \mathrm{~mm}$ (width) $\times 50 \mathrm{~mm}$ (length) $\times 6 \mu \mathrm{m}$ (thickness) at a stretching rate of $50 \mathrm{~mm} \mathrm{~min}^{-1}$ by use of INTESCO. The uniaxially stretched PET films were prepared by stretching unstretched PET films at $110^{\circ} \mathrm{C}$. The thermal shrinkage test was carried out by measuring the sample length at room temperature before and after annealing for $10 \mathrm{~min}$ at $110^{\circ} \mathrm{C}$. The samples for thermal shrinkage test were prepared by cutting the film into strips in different directions. The 
angular dependence of polarized infrared absorbances was measured for the sample rotated at different angles around its central axis normal to the sheet plane under the fixed direction of the electric vector of the incident infrared rays.

\section{RESULTS AND DISCUSSION}

Figure 2 shows that an unstretched PET film gives an almost circular pattern in the angular dependence of transmitted microwave intensity. This circular pattern reflects a random orientation of molecular chains in the plane of the film. X-Ray diffraction and mechanical and infrared measurements on this unstretched PET all indicated random orientation of molecules.

On the other hand, a uniaxially stretched PET film gives an orientation pattern like an ovaloid, as can be seen in Figure 2. The stretching axis corresponds to MD. The transmitted microwave intensity is the lowest in MD, while it is the highest in TD. In other words, the attenuation in microwave intensity is the largest in MD. The pattern for the uniaxially stretched PET film suggests that molecules are arranged on an average in MD.

In Figure 3, $I_{\max } / I_{\min }$ is plotted against draw ratio of a film uniaxially stretched at $110^{\circ} \mathrm{C}$. It increases with increasing draw ratio. Here, the values at $150 \mu \mathrm{m}$ thickness were used for the ratio $I_{\max } / I_{\min }$. The orientation pattern changed from a circular to oval type as the draw ratio was increased.

$\mathrm{X}$-Ray, infrared, birefringence and mechanical measurements showed an increase in the degree of orientation in MD with increasing draw ratio. Therefore, the above results from the microwave method show that the degree of orientation of molecules increases with increasing $I_{\max } / I_{\min }$.

Figures 2 and 4 also show the angular dependence of transmitted microwave intensity at $4.0 \mathrm{GHz}$ for biaxially stretched PET in 20 sheets with $6 \mu \mathrm{m}$ thickness. The direction of the maximum attenuation which corresponds to the direction of the main axis deviates by 39 degree from MD of the film. The ratio of the maximum to minimum intensities was 4.17 and the ratio of the transmitted microwave intensity in TD to that in MD was 1.45 . These ratios can also be taken as a measure of the degree of orientation.

Figure 5 shows the angular dependence of the absorbance at $972 \mathrm{~cm}^{-1}$ in the infrared spectrum for biaxially stretched PET with a thickness of $6 \mu \mathrm{m}$. The absorption band at $972 \mathrm{~cm}^{-1}$ originates from the crystalline phase $^{14}$ and is assigned to the vibrational

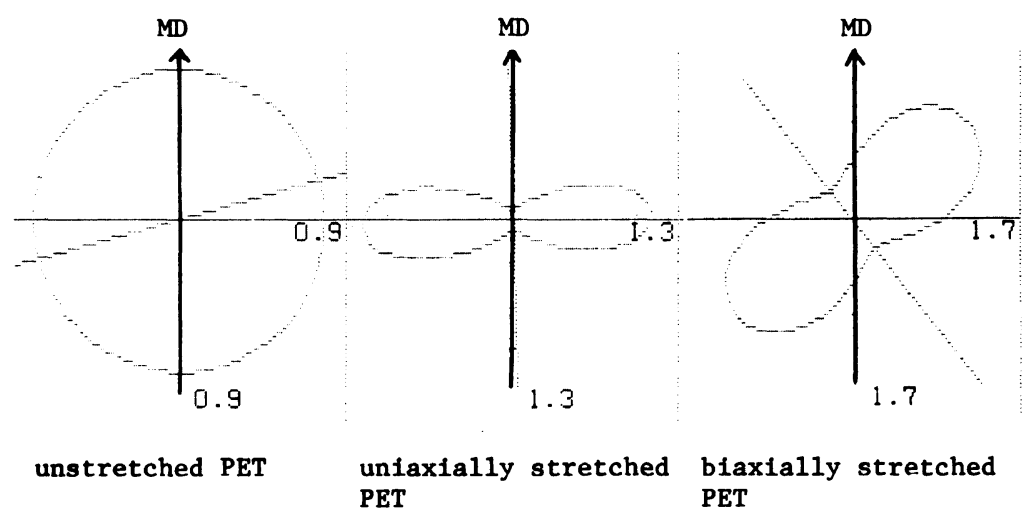

Figure 2. Angular dependence of transmitted microwave intensity at a frequency of $4.0 \mathrm{GHz}$ for unstretched and uniaxially and biaxially stretched poly(ethylene terephthalate) films: MD, machine direction. 
mode with the transition dipole parallel to the chain axis. From this figure the average orientation angle of the molecular chains was estimated to be $c a .37$ degree. The angular dependence of the absorbance at $1025 \mathrm{~cm}^{-1}$ as-

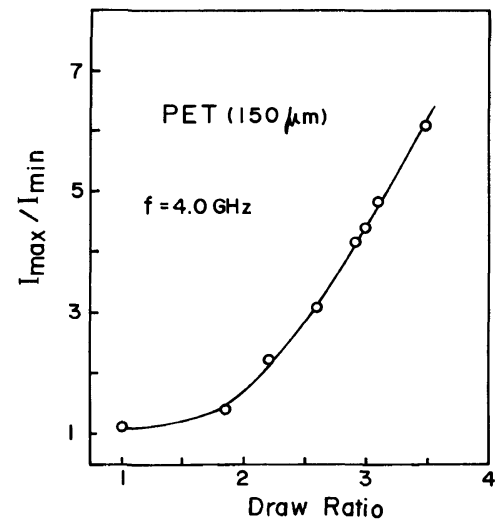

Figure 3. Relationship between draw ratio and the maximum to minimum ratio (at a thickness of $150 \mu \mathrm{m}$ ) of the transmitted microwave intensities measured at a frequency of $4.0 \mathrm{GHz}$ and room temperature for poly(ethylene terephthalate) films (stretched uniaxially at $110^{\circ} \mathrm{C}$.

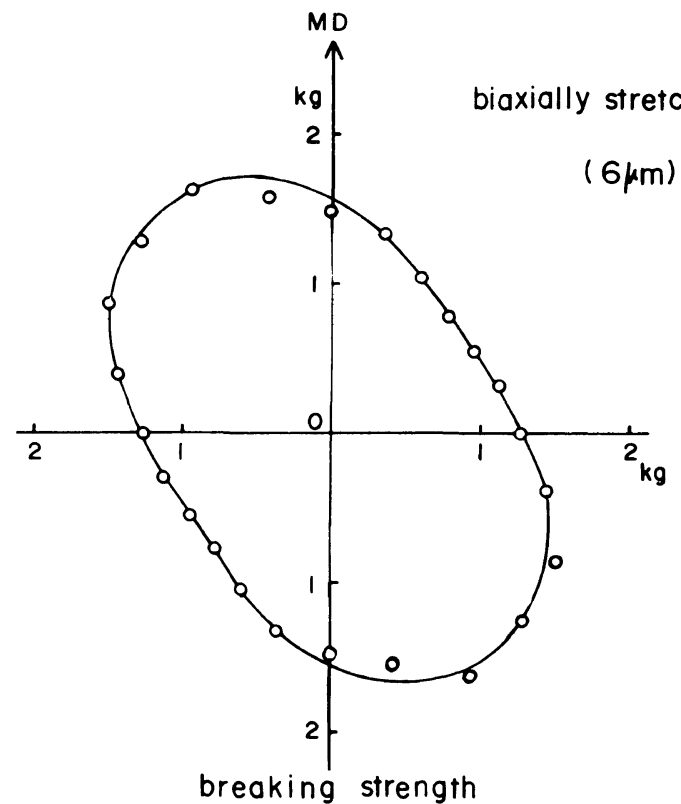

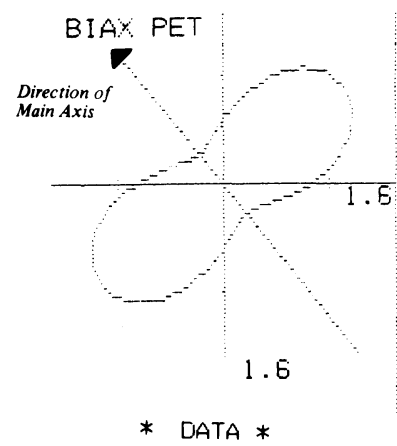

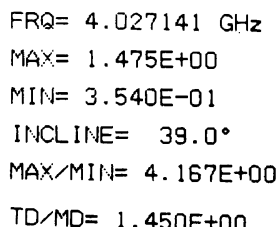

Figure 4. Angular dependence of transmitted microwave intensity at a frequency of $4.0 \mathrm{GHz}$ for biaxially stretched poly(ethylene terephthalate) with a thickness of $120 \mu \mathrm{m}$ : FRQ, measuring frequency; MAX, maximum of transmitted microwave intensity; MIN, minimum of transmitted microwave intensity; INCLINE, orientation angle of main axis of molecular chains; MAX/MIN, ratio of maximum to minimum transmitted microwave intensities; TD/MD, ratio of transmitted microwave intensities in TD to MD.

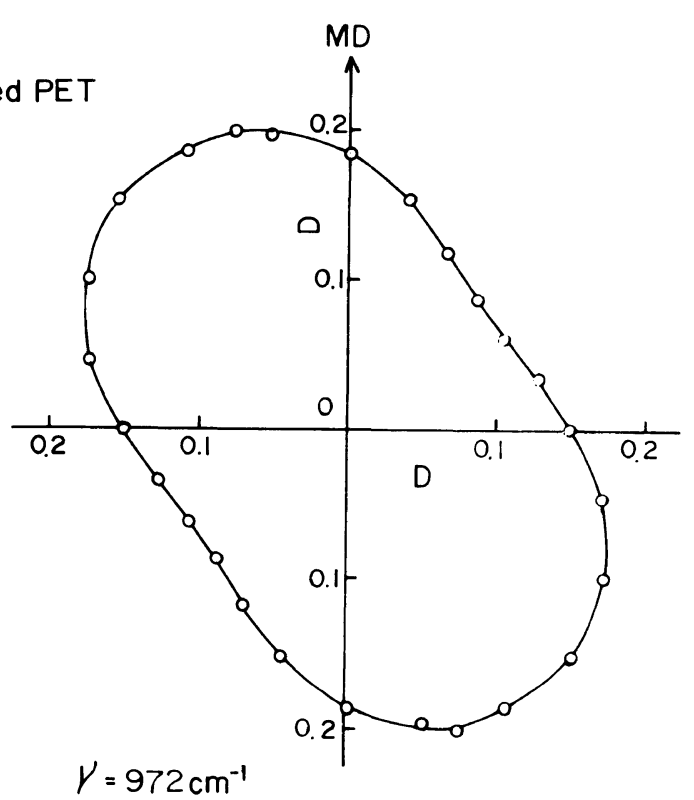

Figure 5. Angular dependences of the absorbance in the polarized infrared spectrum at a wavenumber of $972 \mathrm{~cm}^{-1}$ and that of mechanical breaking strength for the biaxially stretched poly(ethylene terephthalate) with a thickness of $6 \mu \mathrm{m}$ : sample size for stretching, $15 \mathrm{~mm}$ (width) $\times 50 \mathrm{~mm}$ (length); stretching speed, $50 \mathrm{~mm} \mathrm{~min}^{-1}$. 


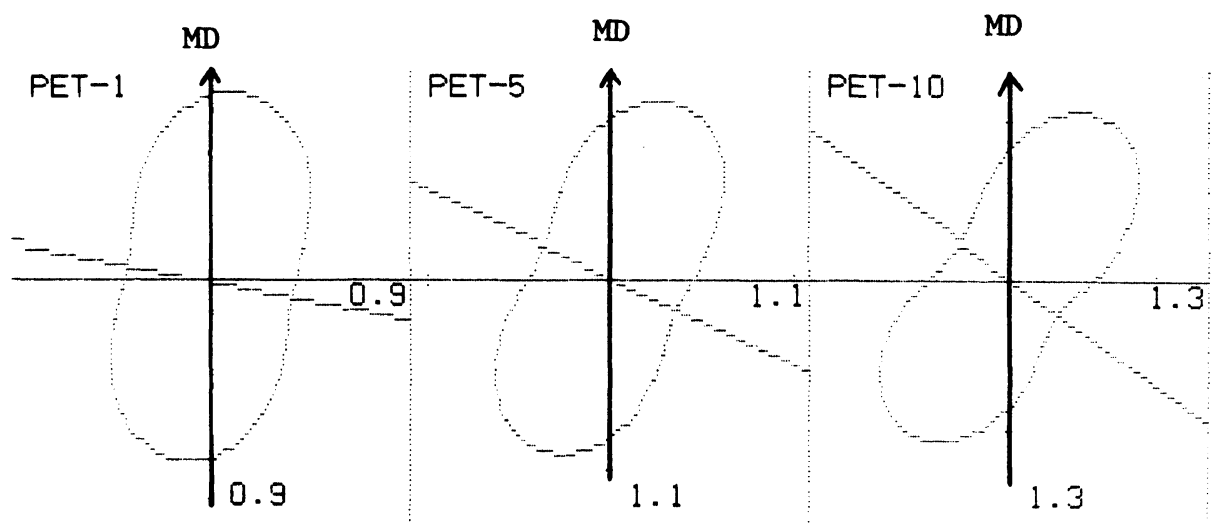

Figure 6. Angular dependences of transmitted microwave intensity at three different positions (PET-1, sample number 1; PET-5, sample number 5; PET-10, sample number 10) in the transverse direction of a biaxially stretched poly(ethylene terephthalate) film with a thickness of $75 \mu \mathrm{m}$ and a width of $1200 \mathrm{~mm}$. The samples are numbered from 1 to 10 from left to right side along the TD.

signed to the amorphous phase ${ }^{15}$ containing crystalline phase also showed similar orientation angle of about 40 degree. These orientation angles correspond well to that determined by microwave method. The direction of the lowest intensity (largest attenuation) agrees with that of the molecular chain orientation. The orientation functions were determined to be 0.064 at $972 \mathrm{~cm}^{-1}$ and 0.049 at $1025 \mathrm{~cm}^{-1}$ from the ratio of absorbances in the parallel to perpendicular directions. On the other hand, the orientation functions $f_{\mathrm{m}}$ were also determined to be 0.208 at $972 \mathrm{~cm}^{-1}$ and 0.195 at $1025 \mathrm{~cm}^{-1}$ from the maximum to minimum ratio in the angular dependences of the absorbances.

As can be seen in Figure 5, the angular dependence of mechanical breaking strength for the biaxially stretched PET film also supports the orientation of molecular chains in a fixed direction. The direction of the maximum breaking strength deviates from MD by about 35 degree, which is close to those estimated from infrared absorbances and microwave intensities, though the mechanical breaking strength is concerned mainly with the amorphous part, while the infrared absorption bands at $972 \mathrm{~cm}^{-1}$ and $1025 \mathrm{~cm}^{-1}$ reflect the crystalline and amorphous phases, respective- ly. The angular dependence of refractive index determined in this work showed that the direction of the maximum refractive index corresponding to the direction of molecular chains coincides well with that found by the microwave method. This agreement indicates that the direction of the largest attenuation in microwaves corresponds to that of the main axis of molecules.

Figure 6 shows orientation patterns at three different positions (PET-1, sample number 1; PET-5, sample number 5; PET-10, sample number 10) in TD of biaxially stretched PET with a $75 \mu \mathrm{m}$ thickness and $1200 \mathrm{~mm}$ width. The pattern differs at the different position of the film sheet. The orientation angles and the maximum to minimum ratio of transmitted microwave intensities at 10 different positions in TD for the same PET film with a $1200 \mathrm{~mm}$ width are shown in Figure 7. The orientation angle decreases with a change in position from the left to right sides, while the maximum to minimum ratio increases. In this way, the anisotropic patterns depend on the machine conditions in preparing films. These findings imply that PET molecules at different positions in the film sheet are directed on an average in different directions with different degree of orientation. Though not shown here, 

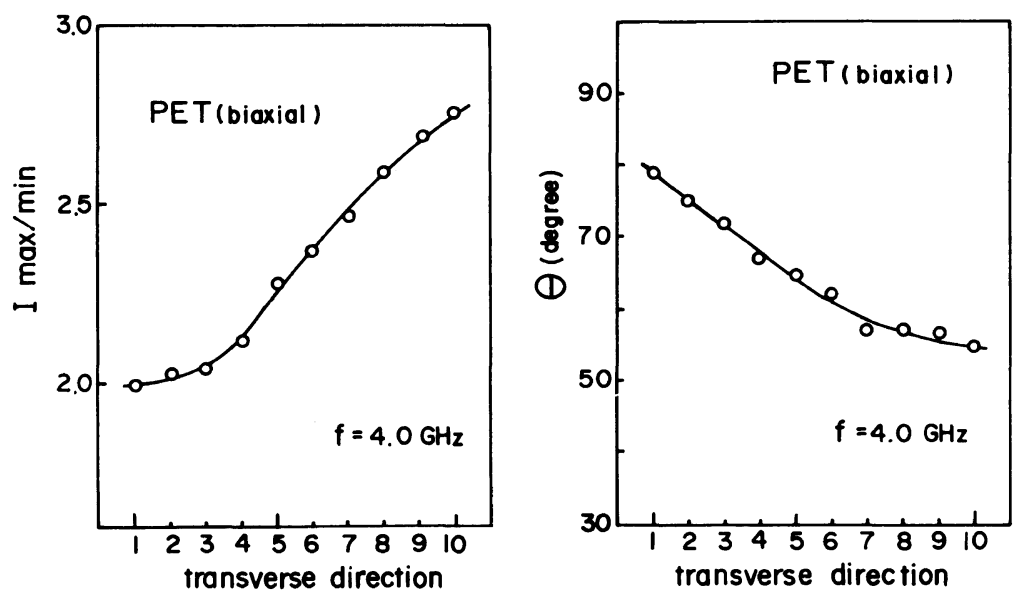

Figure 7. Orientation angles and maximum to minimum ratios of transmitted microwave intensities at many different positions in TD for a biaxially stretched poly(ethylene terephthalate) film with a thickness of $75 \mu \mathrm{m}$. The samples are numbered from 1 to $10 \mathrm{from}$ left to right side along the TD.

the orientation patterns taken at different positions in the same direction of MD were almost the same. The patterns in TD for the biaxially stretched PET film showed that the film uniform in TD could not be prepared. Such non-uniformity in a film may have some effects on its thermal shrinkage and dimensional stability at high temperatures. We therefore measured the thermal shrinkage of the film after annealing for $10 \mathrm{~min}$ at $110^{\circ} \mathrm{C}$. Figure 8 shows the angular dependence of the degree of thermal shrinkage $S$ for the same biaxially stretched PET-1 film described in Figure 6 . Here, $S$ is defined by

$$
S=-\left(L-L_{0}\right) / L_{0}
$$

with $L$ and $L_{0}$ being the length of the strip sample after annealing and the initial length of the sample, respectively. The direction of the maximum shrinkage deviates from $\mathrm{MD}$ by about 15 degree. This direction may be considered roughly perpendicular to the direction of orientation angle determined by the microwave method. The maximum to minimum ratio of thermal shrinkage increased with the change in sample number from 1 to 10 . This result corresponds to that for $I_{\max } / I_{\min }$ ratio shown in Figure 7. Thus, the anisotropic pat-

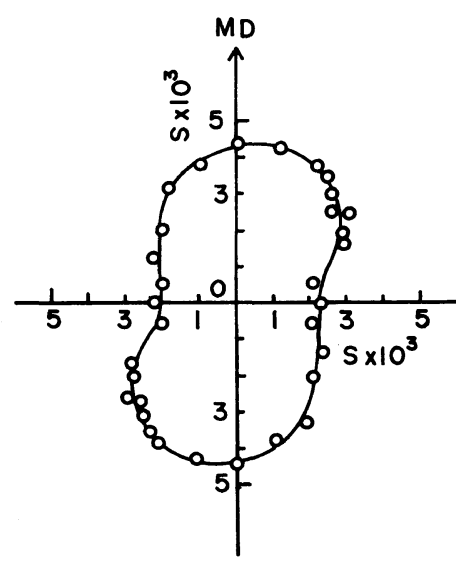

Figure 8. Angular dependence of thermal shrinkage $S$ for biaxially stretched PET-1 (sample number 1) with a thickness of $75 \mu \mathrm{m}$ after annealing the film for $10 \mathrm{~min}$ at $110^{\circ} \mathrm{C}$.

tern in the thermal shrinkage is considered to have some correlation with the orientation pattern obtained by the microwave method. It should be noted here that the biaxially stretched PET films used in this study show the thermal shrinkage in all the directions of the sample, being quite different from the thermal behavior of the uniaxially stretched PET films, which shrink along the MD and expand in TD. 


\section{DISCUSSION}

The orientation angle determined from the microwave method does not depend on sample thickness while the maximum to minimum ratio of transmitted microwave intensity depends on it. Usually, the maximum to minimum ratio should be compared at a constant thickness for different samples. The orientation angle by the microwave method corresponds qualitatively to the results by the mechanical and infrared methods. Thus, the microwave method is useful for determining the direction of molecular orientation. The maximum to minimum ratio of transmitted microwave intensity can be used as an index of anisotropy. At the present stage, however, it is very difficult to derive an anisotropic index independent of sample thickness by use of the present microwave method.

The new method developed here should be applicable to various types of samples, for example, many kinds of tapes, either uniaxially oriented or biaxially oriented, and allows measurement to be completed in as short a time as ca. $30 \mathrm{~s}$ without contact and destruction. With accuracy, precision, and rapidness of measurements kept, this method lends itself to measure a number of samples. Thus, we may use it for quality control by examining machine conditions in preparing films and by taking data on the orientation angle and the maximum to minimum ratio of transmitted microwave intensities in TD of films. Actually, the orientation patterns obtained by the microwave method can be related closely to the dimensional stability and the thermal shrinkage of films at high temperatures. Thus, the microwave method is also useful for studying such properties of films. Furthermore, this method is applicable to thick and opaque films for which the determination of molecular orientation has been quite difficult.

As stated in the section of PRINCIPLE, the resonance curve obtained in the cavity re- sonator system is a function of dielectric constant and dielectric loss. Therefore, the experimentally obtained orientation patterns are expected to be interpreted reasonably in terms of the anisotropy of the dielectric relaxation due to the local motion of molecular chains. In other words, we are required to obtain the informations on the kind of phase (amorphous and/or crystalline phases) and the motional mode of molecular chains attributing such dielectric relaxations, in order to clarify the orientational behavior obtained by the present microwave method. At the present stage, however, it may be rather difficult to make a definite assignment of the molecular motions at microwave frequencies in contrast with the cases of the X-ray diffraction and infrared spectroscopy.

Acknowledgements. The author is grateful to Prof. Masamichi Kobayashi and Dr. Kohji Tashiro of Osaka University for their helpful comments.

\section{REFERENCES}

1. I. M. Ward, "Structure and Properties of Oriented Polymers," Applied Science, London, 1975.

2. I. M. Ward, "Development in Oriented Polymers-1," Applied Science, London, 1982.

3. S. Osaki, TAPPI J., 70, 105 (1987).

4. K. Yamafuji and Y. Ishida, Kolloid-Z.Z. Polym., 183, 15 (1962).

5. Y. Ishida, J. Polym. Sci., A-2, 7, 1835 (1969).

6. T. M. Shaw and J. J. Windle, J. Appl. Phys., 21, 956 (1950).

7. K. K. S. Jamawl and A. Dhar, Rev. Sci. Instrum., 52, 767 (1981).

8. N. G. McGraw, R. E. Reed, and G. Williams, "Anelastic and Dielectric Effects in Polymeric Solids," John Wiley, New York, N. Y., 1967.

9. S. Osaki, S. Uemura, and Y. Ishida, J. Polym. Sci., $A-2,9,585$ (1971).

10. S. Osaki and T. Kotaka, Ferroelectrics, 32, 1 (1981).

11. S. Osaki and Y. Ishida, J. Polym. Sci., A-2, 12, 1727 (1974).

12. H. Kakutani, J. Polym. Sci., A-2, 8, 1177 (1970).

13. E. L. Ginzton, "Microwave Measurements," McGraw-Hill, New York, N. Y., 1957.

14. W. H. Cobbs, Jr., and R. L. Burton, J. Polym. Sci., 10, 275 (1953).

15. A. Miyake, J. Polym. Sci., 38, 479 (1959). 